\title{
THE GROUP OF AUTOMORPHISMS OF A 3-GENERATED 2-GROUP OF INTERMEDIATE GROWTH
}

\author{
R. I. GRIGORCHUK AND S. N. SIDKI
}

We decidate this paper to John Rhodes on his 65th birthday.

\begin{abstract}
The automorphism group of a 3-generated 2-group $G$ of intermediate growth is determined and it is shown that the outer group of automorphisms of $G$ to be an elementary abelian 3- group of infinite rank.
\end{abstract}

\section{INTRODUCTION}

The study of automorphism groups of structures, algebraic or otherwise, is an established classification procedure in mathematics. Computing the group of automorphisms of a particular structure requires deep understanding of multiple aspects of the structure, of its substructures and of its quotients.

The object of this paper is to describe the group of automorphism of the 3generated infinite 2-group $G(2)$ constructed by the first author and shown to have intermediate growth; see [?],[?]. The approach is similar but differs in many details to the one followed by the second author in determining the group of automorphisms of $G(3)$, the 2-generated infinite 3-group constructed by him and Gupta; see [?], [?]. It has been possible to answer many question concerning these groups thanks to the fact they are defined to act on regular one-rooted trees, using easy recursion. The accessibility of such groups to detailed study has opened up a new experimental field in infinite group theory. Since then investigations of groups acting on regular trees have grown in various directions, such as that of branch groups [?] and of groups of finite automata [?],[?].

The groups $G(2), G(3)$ have trivial centers and therefore are isomorphic to their inner group of automorphisms. More importantly though, their automorphisms are induced by conjugation in the full group of automorphisms of the respective trees on which they act. This is an aspect of rigidity which was first proven for the group $G(3)$ and was then shown in [?] to hold for branch groups with some additional natural conditions. A further development along this line appears in [?] where it proven that the branch action on the associated trees to be unique for a wide class of branch groups,

The main result of [?] is that the group of automorphisms of $G(3)$ is a $\{2,3\}$ torsion group which splits as

$$
\operatorname{AutG}(3)=(\operatorname{Inn} G(3))(V D),
$$

1991 Mathematics Subject Classification. Primary 20F05, 20F10.

Key words and phrases. Burnside groups, automorphisms of groups, just-infinite groups, intermediate growth .

The first author acknowledges support from NSF grant DMS-0308985 and the second author acknowledges support from the Brazilian scientific agency CNPq. 
where $V$ is an elementary abelian 3-group of infinite rank and $D$ is elementary abelian of order four which normalizes $V$.

We will show in this paper that the group of automorphisms of $G(2)$ is a 2-group. More precisely. we will prove

Theorem 1. The group $G(2)$ admits an infinite set of automorphisms of order 2 which generate a group $V$ such that AutG(2) $=(\operatorname{Inn} G(2)) V$ and OutG(2) is elementary abelian 2-group of infinite rank.

\section{Preliminaries}

2.1. The binary tree and its automorphisms . The binary tree, denoted by $T$, has as its vertices all finite strings $u$ with entries 0,1 , with the ordering defined by $v<u$ provided $u$ is an initial sequence of $v$. Each vertex $u$ is the root of a subtree $T_{u}$ whose vertices are all strings with prefix $u$. Let $\mathcal{A}$ be the group of automorphisms of $T$ and let $a$ be the automorphism of $T$ which interchanges the vertices $0 u$ and $1 u$ for each string $u$. Using the natural isomorphism between $T$ and its subtrees $T_{0}, T_{1}$ we may represent any $x \in \mathcal{A}$ as $x=\left(x_{0}, x_{1}\right) a^{i}$, where $x_{0}, x_{1} \in \mathcal{A}$ and $i \in\{0,1\}$. In this manner the group $\mathcal{A}$ is identified naturally with the wreath product $\mathcal{A} w r C_{2}$ of $\mathcal{A}$ by the cyclic group $C_{2}$ of order two. Thus, in this representation, the identity element $1 \in \mathcal{A}$ is $1=(1,1) a^{0}$. The automorphisms $x_{0}, x_{1}$ have similar 'developments' in terms of certain $x_{00}, x_{01}, x_{10}, x_{11} \in \mathcal{A}$, and so on.

Given $x \in \mathcal{A}$, then define the following elements of $\mathcal{A}: x^{(0)}=x, x^{(1)}=(x, x)$ and recursively $x^{(i)}=\left(x^{(i-1)}\right)^{(1)}$ for all $i \geq 1$.

The $n$th level stabilizer $s_{H}(n)$ of a subgroup $H$ of $\mathcal{A}$ is the kernel of the action of $H$ on the strings of length $n$. For every string $u$ of length $n$, the elements of $s t_{\mathcal{A}}(n)$ induce automorphisms of the tree with root $u$; thus we obtain the projection map $\pi_{u}: s t_{\mathcal{A}}(n) \rightarrow \mathcal{A}$. Given a subgroup $H$ of $\mathcal{A}$, for each vertex $u$ we write $u * H$ for the subgroup of $\mathcal{A}$ which induces $H$ on the subtree $T_{u}$ and acts trivially outside this subtree. The group generated by all subgroups $u * H$ with $u$ of length $n$ is of course the direct product of these subgroups and we denote it by $\times_{2^{n}} H$. Any subgroup or element of $H$ outside $s t_{H}(1)$ is called active.

For any subgroup $H$ of $\mathcal{A}$, the rigid stabilizer rist $_{G}(u)$ of $u$ in $G$ is the subgroup $H \cap(u * \mathcal{A})$. For each $n \geq 0$ we write $\operatorname{rist}_{H}(n)$ for the group generated by all subgroups rist $_{H}(u)$ with $u$ of length $n$.

A subgroup $H$ of $\mathcal{A}$ is called a branch group if for all $n$ if it acts transitively on the set of $n$th level vertices (that is, the vertices which are strings of length $n$ ) and if $\operatorname{rist}_{H}(n)$ has finite index in $G$. As was proven in [?], a branch group is just infinite provided rist $_{G}(u)$ has finite abelianization.

2.2. The group $G(2)$. The group $G=G(2)$ is generated by the three automorphisms $a, b, c$ of the tree $T$ where $b, c$ are defined recursively by

$$
b=(a, c), c=(a, d), d=(1, b) ;
$$

note that $d=b c$ and $a^{2}=b^{2}=c^{2}=d^{2}=1$. In this section we will give a summary of some known facts about $G$ (see: [?], [?]) and will prove some new ones which will be necessary to reach our main result. 
Denote by $H$ the 1 st level stabilizer $s t_{G}(1)$. Then $H$ is a normal subgroup of index 2 in $G$ and is generated by the four elements

$$
c, d, c^{a}=(d, a), d^{a}=(b, 1) ;
$$

the symbol $x^{y}$ denotes $y^{-1} x y$, the conjugate of $x$ by $y$. Thus, $H$ is a subdirect product of $G \times G$ and $G$ is a subgroup of $(G \times G)<a>$.

2.2.1. Length and Depth Functions. Let $|g|$ denote the length of $g$ with respect to the system of generators $a, b, c, d$. Any shortest word representing $g$ has the form

$$
* a * a \ldots * a *
$$

where $*$ denotes an element from the set $\{b, c, d\}$, yet the first and last $*$ can be the empty word. Let $g \in G$ and write its representation on the tree as $g=$ $\left(g_{0}, g_{1}\right) a^{\varepsilon}, g_{0}, g_{1} \in G, \varepsilon=0,1$. Then $\left|g_{i}\right| \leq \frac{1}{2}(|g|+1)$. If the first or last $*$ is empty then the inequality improves to $\left|g_{i}\right| \leq \frac{1}{2}|g|$; see [?].

There is a level $k$ of the tree such that in the development of $g$, its states $g_{u} \in$ $\{1, a, b, c, d\}$ for all strings $u$ of length $k$. The smallest such $k$ is called the depth of $g$ and is denoted by $\operatorname{depth}(g)$. From the inequalities for the length function we get $\operatorname{depth}(g) \leq \log _{2}(|g|)+1$.

Proposition 1. The group $G$ is an infinite 2-group.

The proof follows simply by induction on the word length.

2.2.2. Special subgroups. (i) From the definition of the generators, $b=(a, c), c=$ $(a, d), d=(1, b)$, we calculate

$$
\begin{aligned}
& (a b)^{2}=(c a, a c),(a c)^{2}=(d a, a d),(a d)^{2}=(b, b)=b^{(1)}, \\
& (a c)^{4}=b^{(2)},
\end{aligned}
$$$$
(a b)^{8}=b^{(3)} \text {, }
$$$$
\left[b, b^{(2)}\right]=(1,([a, b], 1)) \text {. }
$$

(ii) We have the dihedral groups:

$\langle a, b\rangle$ of order $32,\langle a, c\rangle$ of order $16,\langle a, d\rangle$ of order 8 .

(i) Let $B, C, D$ be the normal closures in $G$ of $\langle b\rangle,\langle c\rangle,\langle d\rangle$, respectively. Then $G$ factorizes as the following semidirect products

$G=C<a, d\rangle=D<a, c\rangle=B<a, d\rangle$.

(iii) Rigid stabilizers. The 1st restricted stabilizer is rist $_{G}(1)=D=B \times B$.

Define $K=<(a b)^{2}>^{G}$. Then, $K=[B, G],[D, H]=K \times K$ and the quotient group $G / K=K\left\langle a, d>+K\langle b\rangle\right.$ is finite of order 16 . Since $\left[(a b)^{2}, d\right]=$ $\left(1,(a b)^{2}\right)$, it follows that

$K=(K \times K)<(a b)^{2}>$ and thus, $[K, D[=K \times K$. Inductively we produce the series $G>K>K \times K>\ldots>\times_{2^{m}} K>\ldots$ of normal subgroups of $G$. It is clear that rist $_{G}(m) \geq \times_{2^{m}} K$ for $m \geq 1$.

Proposition 2. The group $G$ is just-infinite and not abelian-by-finite.

Proof. Since $G$ is a torsion branch group and $\operatorname{rist}_{G}(m)$ has finite index in $G$, it follows that $G$ is just-infinite. Also, $G$ is not abelian-by-finite, for otherwise it would finite.

We note that $(a b)^{8}=(b)^{(3)} \in K$ implies that $(b)^{(i+3)} \in \times_{2^{m}} K$ for all $i$. Also, from $(a c)^{2}(d a)^{2}=\left((a b)^{2}\right)^{c a}$ we conclude $(a c)^{2} \equiv(a d)^{2}$ modulo $K,(a c)^{4} \equiv(a b)^{4}$ modulo $K \times K$. 
(iv) The group $G$ has 7 subgroups of index 2 (see [?]). These are:

$J(0,2)=\left\langle a, b>^{G}=\left\langle a, a^{c}, b>\right.\right.$,

$J(0,3)=\left\langle a, c>^{G}=\left\langle a, a^{d}, c>\right.\right.$,

$J(0,4)=<a, d>^{G}=<a, a^{b}, d>$,

$J(0,5)=<b, a c>^{G}=<b, a c>$,

$J(0,6)=<c, a b>^{G}=<c, a b>$,

$J(0,7)=<d, a b>^{G}=<d, a b>$,

$J(1,2)=H=s t_{G}(1)=<c, c^{a}, d . d^{a}>=D<c, c^{a}>$.

The group $J(0, i) \cap H$ is a subdirect product of $G \times G$ for $i=2,3,5,6$, and $J(0, i) \cap H$ is a subdirect product of $J(0,5) \times J(0,5)$ for $i=4,7$. Furthermore, $B$ is a subdirect product of $J(0,3) \times J(0,3)$, and $C$ is a subdirect product of $J(0,4) \times J(0,4)$. The first three groups in the list are not 2 -generated, the next three are not cyclic and the last one which the group $H$ is not 3-generated. Therefore, $H$ is a characteristic subgroup.

2.2.3. Centralizers. (i). If $N$ be a normal non-trivial subgroup of $G$, then $C_{G}(N)$ is trivial. Otherwise, $N \cap C_{G}(N)=Z(N)$ has finite index in $G$ and thus $G$ is solvable and finite; a contradiction.

(ii) Suppose $X \leq B$ is nontrivial such that $U=X \times\{1\}$ is normal in $H$. It is clear that $C_{G}(U) \leq H$. Let $(w, v)$ commute with $U$, then $w$ commutes with $X$ and therefore $w=1$. Thus, $C_{H}(U)=1 \times B$.

(iii) Let $W, V$ be commuting normal subgroups of $H$. Then, $W \leq B \times\{1\}, V \leq$ $\{1\} \times B$ or vice versa:

if $W \cap(B \times\{1\})$ is trivial then $W$ is contained in the centralizer of $B \times\{1\}$ and therefore is contained in $\{1\} \times B$; if $W \cap(B \times\{1\})=X \times\{1\}$ is non-trivial then, by (ii), its centralizer is equal $\{1\} \times B$ and therefore $V \leq\{1\} \times B$.

(iv) The centralizer of $a$ in $G$ factorizes as $C_{G}(a)=\{(v, v) \mid v \in B\}<(a c)^{4}><$ $a>$. For, $C_{G}(a)=C_{H}(a)<a>$ and since $H=(B \times B)\left\langle c, c^{a}\right\rangle$, given $x \in C_{H}(a)$, there exist $u, v \in B, i, j \geq 0$ such that $x=(u, v)\left((a d)^{i} a^{j},(d a)^{i} d^{j}\right)$ and $u(a d)^{i} a^{j}=v(d a)^{i} d^{j}$..

Proposition 3. ([?]) The centralizer $C_{G}(a)$ is finitely generated and if an element $g \in G$ has finitely generated centralizer, then $g$ is conjugate to a.

2.2.4. Decomposable groups. (i) The following result about groups decomposed as sums of indecomposable subgroups implies that their automorphisms permute the indecomposable factors.

Proposition 4. Let $U$ be a group with trivial center which decomposes as $U=$ $U_{1} \oplus U_{2} \oplus \ldots \oplus U_{s}=W_{1} \oplus W_{2} \oplus \ldots \oplus W_{t}$ where $U_{i}, W_{j}$ are indecomposable normal subgroups of $U$. Then, $\left\{U_{1}, U_{2}, \ldots, U_{s}\right\}=\left\{W_{1}, W_{2}, \ldots, W_{t}\right\}$ and $s=t$.

Proof. Let $p_{i}$ be the projection of $U$ onto $U_{i}$. Then $<p_{i}\left(W_{j}\right) \mid 1 \leq j \leq t>=$ $U_{i},\left[p_{i}\left(W_{j}\right), p_{i}\left(W_{k}\right)\right]=1$ for $j \neq k$ and as $U_{i}$ has trivial center, $U_{i}=p_{i}\left(W_{1}\right) \oplus$ $p_{i}\left(W_{2}\right) \oplus \ldots \oplus p_{i}\left(W_{t}\right)$. Since $U_{i}$ is indecomposable, there exists a unique $j=f(i)$ such that $U_{i}=p_{i}\left(W_{j}\right)$ and $p_{i}\left(W_{k}\right)=1$ for all $k \neq j$. Thus $f$ is injective and by the symmetry between the two decompositions of $U$ we get that $f$ is bijective and thus, $s=t$. We may rearrange indices so that $f$ is the identity function and so $U_{i}=p_{i}\left(W_{i}\right), p_{i}\left(W_{k}\right)=1$ for all $k \neq i$. Hence, $U_{i}=p_{i}\left(W_{i}\right)=W_{i}$ for all $i$. 
(ii) The group $G=G(2)$ is indecomposable. For, suppose $G=U+V$ for some subgroups $U, V$. Then $U \cap V$ is a central subgroup and is therefore trivial. Hence either $U$ or $V$ has finite order and is therefore trivial.

(iii) Let $R$ be subgroup of $H$ which projects onto $G$ in both coordinates. Then $R$ is indecomposable. For, suppose $R=U+W$. Then $G=\pi_{i}(U)+\pi_{i}(W)$ and so either $\pi_{i}(U), \pi_{i}(W)$ is trivial. Say $\pi_{0}(U)=1, \pi_{0}(W)=G$ then $\pi_{1}(U)=G$ and $\pi_{1}(W)=1$; thus, $G \times G \leq R$ and $R=H$. It follows that $H=G \times G$. and $G$ is infinitely generated; a contradiction.

(iv) The subgroups $H$ and $J(0, i) \cap H$ are indecomposable for $i=2,3,5,6$ by (iii) and 2.2.2.

(v) Let $M$ be any one of the maximal subgroups $J(0, i)$ for $i=2,3,5,6$. Then $M$ is indecomposable. Suppose the contrary and let $M=U+W$ for some $U, W \leq$ $M$. If $U \leq H$ then $M \cap H=U+(W \cap H)$ and by (iii), $W \cap H=1$; thus $W$ has order 2; a contradiction. Hence $U$ and $W$ are not contained in $H$. Now let $U_{H}=U \cap H, W_{H}=W \cap H$. We consider the intersection of $U_{H}$ and $W_{H}$ with $K \times K$. Now $K \times K \leq M \cap H$ and is normal in $H$. Let $U_{H} \cap(K \times 1)=\dot{K} \times 1$. If $\dot{K} \neq 1$ then $C_{G}(\dot{K} \times 1) \geq W$; but this absurd since $W$ is active.. Thus $\dot{K}=1$. Now, since $U_{H}$ and $K \times 1$ are both normal in $M$, it follows that $U_{H}$ centralizes $K \times 1$ and $\pi_{0}\left(U_{H}\right)$ centralizes $K$. Since $K$ is a normal subgroup of $G$, it follows that $\pi_{0}\left(U_{H}\right)=1$. By symmetry, we have $\pi_{1}\left(U_{H}\right)=1$. Hence $U_{H}=1, o(U)=2$ and $U^{G}$ is a finite group; a contradiction.

(vi) The subgroup $B$ is indecomposable. This is so since $B$ is a subdirect product of $J(0,3) \times J(0,3)$ and a decomposition of $B$ induces a decomposition of $J(0,3)$.

(vii) The subgroup $D$ is characteristic in $G$ : the image of $D=$ rist $_{G}(1)=B \times B$ under an automorphism of $G$ is a subgroup of $H$ decomposable as a direct sum of two normal subgroups of $H$ and so, the result follows by an application of item (iii) of 2.2 .3 .

\section{Proof of the main Result}

Let $A$ denote $A u t(G)$, the automorphism group of $G$. Since the center of $G$ is trivial, we identify $G$ with its group of inner automorphisms $\operatorname{Inn}(G)$. By Proposition 3, the conjugacy class of $a$ in $G$ is a characteristic subset of $G$. Therefore, given $\tau \in A$, on composing it with a conjugation by some $h \in H$, we may assume that $\tau$ fixes $a$. Denote the subgroup of automorphisms which fix $a$ by $A_{a}$. Since $D=B \times B$ is a characteristic subgroup of $G$ and $B$ is indecomposable, $\tau$ permutes $\{B \times 1,1 \times B\}$. We may suppose that $\tau$ leaves both $B \times 1,1 \times B$ invariant; if not, then we modify $\tau$ to $\tau a$. Recall $\pi_{0}, \pi_{1}$ the projections of $H$ onto $G$. Then, as $H$ is a characteristic subgroup of $G$, we have that $\tau \pi_{0}, \tau \pi_{1}$ are homomorphisms from $H$ onto $G$. If $h \in H$ is in the kernel of $\tau \pi_{0}$ then $h^{\tau} \in 1 \times B=\operatorname{ker}\left(\pi_{0}\right)$ and so $h \in 1 \times B$ Therefore, for $i=0,1$, the maps $h^{\pi_{i}} \rightarrow h^{\tau \pi_{i}}$ define automorphisms $\tau_{i}$ of $G$. Hence, $\tau$ restricted to $H$ is induced by the automorphism $\left(\tau_{0}, \tau_{1}\right)$ of $G \times G$ and as $\tau$ commutes with $a$, we have $\tau_{0}=\tau_{1}$. We conclude that a general automorphism $\tau$ of $G$ is a restriction of an automorphism of the overgroup $(G \times G)\langle a\rangle$, having the form $(\sigma, \sigma) h a^{i}$ for some $\sigma \in \operatorname{Aut}(G), h \in H, i=0,1$. A unique form $\tau=(\sigma, \sigma) h a^{i}$ can be obtained where $i=0,1$ and $h$ is an element from a fixed right transversal $S$ of $C_{H}(a)=\left\{(u, u) \mid u \in B<(a d)^{2}>\right\}$ in $H$. Since $\sigma$ can be developed similarly we obtain sequences $\tau(m) \in \operatorname{Aut}(G), h(m) \in S$ and $i(m) \in\{0,1\}$ defined by $\tau(0)=\tau, h(0)=h, i(0)=i$ and inductively, $\tau(m)=(\tau(m+1), \tau(m+1)) h(m) a^{i(m)}$. 
This development of $\tau$ shows that for all $k \geq 1$, it is the restriction of an automorphism of $\left(\times_{2^{k}} G\right) P(k)$ to $G$, where $P(k)$ is a Sylow 2-subgroup of $\operatorname{Sym}\left(2^{k}\right)$. Hence, $s t_{G}(k)$ is $\tau$-invariant for all $k \geq 1$. Now, the sequences $h(m), i(m)$ determine the following well-defined infinite product of automorphism of the tree

$$
\alpha=\ldots\left(h(m) \alpha^{i(m)}\right)^{(m)} \ldots\left(h(1) \alpha^{i(1)}\right)^{(1)} h(0) \alpha^{i(0)} .
$$

Clearly, $\alpha$ is an automorphism of the tree, and $g^{-1}(g)^{\alpha \tau^{-1}} \in s t_{G}(k)$ for all $k \geq 1$ and all $g \in G$. As $\cap_{k \geq 1} s t_{G}(k)=1$ we conclude that $\alpha=\tau$. We have shown

Proposition 5. The group $A=\operatorname{Aut}(G)$ is isomorphic to $N_{A u t(T)}(G)$.

3.1. Some outer automorphisms. Define the automorphism of the tree $\theta(0)=$ $(a d, a d)=(a d)^{(1)}$ of $\times{ }_{2} G$ and define for all $i \geq 0, \theta(i)=(a d)^{(i+1)} \in \times_{2^{i+1}} G$. Let $V=\{\theta(i) \mid i \geq 0\}$. We calculate

$[\theta(i), a]=1$

$[\theta(0), c]=\left[(a d)^{(1)}, c\right]=([a d, a],[a d, d])=\left([d, a],[a, d]^{d}\right)=b^{(2)}$,

$[\theta(0), d]=\left[(a d)^{(1)}, d\right]=(1,[a d, b])=\left(1,[a, b]^{d}\right) \in \times{ }_{2} K$

$[\theta(1), c]=([\theta(0), a],[\theta(0), d])=\left(1,\left(1,[a, b]^{d}\right)\right) \in \times_{2^{2}} K$

$[\theta(1), d]=(1,[\theta(0), b])=(1,[\theta(0), c d])=\left(1,[\theta(0), d][\theta(0), c]^{d}\right) \in \times_{2^{2}} K$.

By induction on $i$, we have

$[\theta(i+1), c]=(1,[\theta(i), d]) \in \times_{2^{i+2}} K$

$[\theta(i+1), d]=(1,[\theta(i), b]) \in \times_{2^{i+2}} K$.

Therefore, $\theta(i) \in N(G)$ in $\times_{2^{i+1}} G$. Now, $\theta(0)=(a d)^{(1)} \in \times{ }_{2} G \backslash G$; otherwise, $(a c)^{-2}(a d)^{(1)}=(a d, 1) \in H$ and $a d \in B \leq H$, which is absurd. Also, $\theta^{2}(0)=$ $\left((a d)^{2},(a d)^{2}\right)=b^{(2)} \in G$. Similarly, $\theta(i) \in \times_{2^{i+1}} G \backslash \times_{2^{i}} G$. We have shown that $V \subset N_{A}(G) \backslash G, V^{2} \subset G$ and that modulo $G$, the set of $\theta(i)$ 's are independent.

Since $c^{a} c=(d a, a d) \in G$, the automorphisms $\theta(0)=(a d, a d)$ can be modified modulo $G$ to $\mu=(d a, a d)(a d, a d)=\left(1,(a d)^{2}\right)$ of order 2 . Similarly, we produce the outer automorphisms $\mu(i)=\left(c^{a} c\right)^{(i)} \theta(i)$ for $i \geq 0$ where $\mu(0)=\mu$. It is clear that $\{\mu(i) \mid i \geq 0\}$ generate $V$ modulo $G$.

Lemma 1. Let $y=(g)^{(m)} \in \times_{2^{m}} G, m \geq 1$. Then $y$ normalizes $G$ if and only if $g \in B<a d>$.

Proof. Observe that $(a)^{(m)}$ does not normalize $G$, since

$\left[c,(a)^{(m)}\right]=\left(1,\left(1, . .,\left(1,(d a)^{2}\right)\right) \ldots\right) \notin G$. Also, we know $(b)^{(m)} \in G$ and $\theta(m-$ $1)=(a d)^{(m)}$ normalizes $G$. Now the proof follows from $G=(B<a d>)<a>$.

3.2. The final step. We start with

Lemma 2. Let $(\tau, g)$ be a pair with $\tau \in A, g \in G$ such that $x^{\tau}=x^{g}$ for $x \in\{b, c, d\}$. Then $\tau$ is an inner automorphism.

Proof. We will first prove the assertion for the pair $(\tau, g)$ with $\tau \in A_{a}$. Write $\tau=(\sigma, \sigma) h a^{i}$ in its normal form. Then since $\tau$ fixes $a$, we have $h=\left(h^{\prime}\right)^{(1)}$. Suppose we have a counterexample $(\tau, g)$ with $g$ of minimal length. Then, $|g|=n>1$ and that the minimal word representing $g$ has the form $a * a * a \ldots a *$ where the last * may be empty. It is clear that $g \in H$ if and only $i=0$. The length of $g$ is even; for otherwise, the form of $g$ ends in $a$ and $g$ is active and so $a$ can be cancelled from both $\tau$ and $g$. Then, $b^{\tau}=\left(a^{\sigma h^{\prime}}, c^{\sigma}\right)=b^{g}=\left(a^{g_{0}}, c^{g_{1}}\right)$ and therefore 
$a^{\sigma h}=a^{g_{0}}, c^{\sigma h^{\prime}}=c^{g_{1}}$. Similarly, we obtain $d^{\sigma h^{\prime}}=d^{g_{1}}, b^{\sigma h^{\prime}}=b^{g_{1}}$. Let $\sigma^{\prime}=\sigma h^{\prime} g_{0}^{-1}$ and $g^{\prime}=g_{1} g_{0}^{-1}$. Therefore, the pair $\left(\sigma^{\prime}, g^{\prime}\right)$ satisfies the hypothesis and $\sigma^{\prime}$ fixes $a$.

From 2.2.1, $\left|g_{i}\right| \leq \frac{1}{2}(|g|+1)$ for $i=0,1$ and as $|g|$ is even, $\left|g^{\prime}\right| \leq|g|$. Then $\left|g^{\prime}\right|=|g|$ and since the first coordinate of $d$ is the identity 1 , we conclude that $d$ cannot occur as one of the $*$ symbols in the form of $g$. We repeat the process to the pair $\left(\sigma^{\prime}, g^{\prime}\right)$ producing $\left(\sigma^{\prime \prime}, g^{\prime \prime}\right)$. Then $\left|g^{\prime \prime}\right|=|g|$ and we find that $d$ cannot occur as one of the $*$ symbols in the form of $g^{\prime}=g_{1} g_{0}^{-1}$; therefore $c$ cannot occur as one of the $*$ symbols in the form of $g$. One more repetition of the process eliminates the occurrence of $b$ in the form of $g$ as well and we reach a contradiction.

In the general case, where $\tau$ may not fix $a$, we have $a^{\tau}=a^{y}$ for some $y \in G$ and thus we simply apply the previous case to the pair $\left(\tau y^{-1}, g y^{-1}\right)$.

Since $b^{\tau}, c^{\tau}, d^{\tau}$ have finite depth, there exists a level $m$ such that $\left(b^{\tau}\right)_{u},\left(c^{\tau}\right)_{u},\left(d^{\tau}\right)_{u} \in$ $\{1, a, b, c, d\}$ for all strings $u$ of length $m$. We observe from

$b^{\tau}=\left(a^{\tau(1) h(0)_{0}}, c^{\tau(1) h(0)_{1}}\right)^{a^{i(0)}}, c^{\tau}=\left(a^{\tau(1) h(0)_{0}}, d^{\tau(1) h(0)_{1}}\right)^{a^{i(0)}}, d^{\tau}=\left(1, b^{\tau(1) h(0)_{1}}\right)^{a^{i(0)}}$

that for each level $k$ there exist a string $u$ of length $k$ and $y \in G$ such that $\left\{\left(b^{\tau}\right)_{u},\left(c^{\tau}\right)_{u},\left(d^{\tau}\right)_{u}\right\}=\{b, c, d\}^{\tau(k) y}$. Hence for the level $m$, there exists $y^{\prime} \in G$ such that for $\gamma=\tau(m) y^{\prime}$, we have $\{b, c, d\}^{\gamma}=\{b, c, d\}$. Since $D$ is a characteristic subgroup of $G$, it follows that $d^{\gamma}=d$ and $\{b, c\}^{\gamma}=\{b, c\}$. Now, $\gamma=\left(\gamma_{0}, \gamma_{1}\right)$ for some $\gamma_{0}, \gamma_{1} \in \operatorname{Aut}(G)$. Suppose $b^{\gamma}=c$. Then $b^{\gamma}=\left(a^{\gamma_{0}}, c^{\gamma_{1}}\right)=(a, d)$ and therefore $c^{\gamma_{1}}=d$ which is impossible. Hence, $\gamma$ fixes $b, c, d$. It follows from the previous lemma that $\tau(m)$ is conjugation by some $q \in G$. Since, $\tau=\tau(m)^{(m)} g$ for some $g \in G$, we have $q^{(m)}$ normalizes $G$. By Lemma $1, q \in G V$. This concludes the proof of the main theorem.

\section{REFERENCES}

[1] Ceccherni-Silberstein, T. G., Scarabotti, F., Tolli, F., "The top of the lattice of normal subgroups of the Grigorchuk group", J. of Algebra 246, (2001), 292-310.

[2] Grigorchuk, R. I. , "On Burnside's problem on periodic groups", Functional Anal. Appl. 14 (1980), 41-43.

[3] Grigorchuk, R. I. , "Degrees of growth of finitely generated groups and the theory of invariant means", Izv. Akad. Nauk SSSR 48 (1984), no.5, 939-985.

[4] Grigorchuk, R. I., Just infinite branch groups, In: New horizons in pro-p groups, Birkhauser (M. P. F. du Sautoy, D. Segal and A. Shalev eds) (2000), 121-179.

[5] Grigorchuk, R. I. , Nekrashevych, V. V., Sushchansky, V. I., "Automata, Dynamical systems and Groups", Proceedings of the Steklov Institute of Mathematics (2000), 134-214.

[6] Grigorchuk, R. I. , Wilson, J. S. , "The uniqueness of the action of certain branch groups on rooted trees". To appear in Geomtria Dedicata.

[7] Gupta, N, Sidki, S, "On the Burnside problem for periodic groups", Math. Z. 182 (1983), 385-388.

[8] de la Harpe, P., Topics in geometric group theory. Univ. of Chicago Press (2000).

[9] Lavreniuk, Y., Nekrashevych, V., "Rigidity of branch groups acting on rooted trees", Geometria Dedicata 89 (2002), 159-179.

[10] Rozkhov, A. V. , "Centralizers of elements in a group of tree automorphisms", Russian Acad. Sci. Izv. Math. 43 (1994), 471-492.

[11] Sidki, S., "On a 2-generated infinite 3-group: subgroups and automorphisms", J. of Algebra 110 (1987), 24-55.

[12] Sidki, S., "Automorphisms of one-rooted trees: growth, circuit structure and acyclicity", J. Math. Sci, 100 (2000), 1925-1943. 
Steklov Mathematical Institute, Gubkina street 8, Moscow 117966, Russia

Current address: Department of Mathematics, Texas A\&M University, College Station, TX 77843-3368, USA

E-mail address: grigorch@math.tamu.edu

Departamento de Matemática, Universidade de Brasília, , 70910-900 Brasília-DF, Brazil

E-mail address: sidki@mat.unb.br 